\title{
SOME RESULTS ON WEIGHTED CRITICAL QUASILINEAR PROBLEMS
}

\section{DONGSHENG KANG}

\begin{abstract}
In this paper, we are concerned with a kind of quasilinear elliptic problems, which involves the Caffarelli-Kohn-Nirenberg inequality and critical exponents. By employing variational methods and analytical techniques, the existence of sign-changing solutions to the problem is proved.
\end{abstract}

Mathematics subject classification (2010): 35J25, 35J60, 35B33. method.

Keywords and phrases: solution, elliptic problem, Caffarelli-Kohn-Nirenberg inequality, variational

\section{REFERENCES}

[1] B. AbDellaoui AND I. Peral, The effect of Harnack inequality on the existence and nonexistence results for quasilinear parabolic equations related to Caffarelli-Kohn-Nirenberg inequalities, NoDEA Nonlinear Differential Equations Appl., 14 (2007), 335-360.

[2] R. Assuncao, P. CARRIAO AND O. MiYAGaki, Subcritical perturbations of a singular quasilinear elliptic equation involving the critical Hardy-Sobolev exponent, Nonlinear Anal., 66 (2007), 13511364.

[3] L. Caffarelli, R. Kohn And L. Nirenberg, First order interpolation inequality with weights, Compos. Math., 53 (1984), 259-275.

[4] D. CAO AND P. HAN, Solutions to critical elliptic equations with multi-singular inverse square potentials, J. Differential Equations, 224 (2006), 332-372.

[5] F. CATRINA AND Z. WANG, On the Caffarelli-Kohn-Nirenberg inequalities: sharp constants, existence (and nonexistence), and symmetry of extermal functions, Comm. Pure Appl. Math., 54 (2001), $229-258$.

[6] V. Felli And M. Schneider, Compactness and existence results for degenerate critical elliptic equations, Commun. Contemp. Math., 7 (2005), 37-73.

[7] N. Ghoussoub, Duality and perturbation methods in critical point theory, Combridge University Press, Combridge, UK, 1993.

[8] N. Ghoussoub And C. YuAn, Multiple solutions for quasi-linear PDEs involving the critical Sobolev and Hardy exponents, Trans. Amer. Math. Soc., 352 (2000), 5703-5743.

[9] T. HoriUCHI, Best constant in weighted Sobolev inequality with weights being powers of distance from the origin, J. Inequal. Appl., 1 (1997), 275-292.

[10] D. KANG, Positive solutions to the weighted critical quasilinear problems, Appl. Math. Comput., 213 (2009), 432-439.

[11] D. KANG, Some properties of solutions to the singular quasilinear problem, Nonlinear Anal., 72 (2010), 682-688.

[12] D. KANG, Y. HUANG AND S. LIU, Asymptotic estimates on the extremal functions of a quasilinear elliptic problem, J. South Central. Univ. Natl., 27, 4 (2008), 96-101.

[13] P. L. Lions, The concentration compactness principle in the calculus of variations, the limit case(I), Rev. Mat. Iberoamericana, 1, 1 (1985), 145-201.

[14] P. L. Lions, The concentration compactness principle in the calculus of variations, the limit case(II), Rev. Mat. Iberoamericana, 1, 2 (1985), 45-121.

[15] K. SANDEEP, On the first eigenfunction of a perturbed Hardy-Sobolev operator, NoDEA Nonlinear Differential Equations Appl., 10 (2003), 223-253. 
[16] S. Secchi, D. Smets And M. Willem, Remarks on a Hardy-Sobolev inequality, C. R. Acad. Sci. Paris Sr. I Math., 336 (2003), 811-815.

[17] D. SMETS, Nonlinear Schrödinger equations with Hardy potential and critical nonlinearities, Trans. Amer. Math. Soc., 357 (2005), 2909-2938.

[18] G. TARANTELlo, Nodal solutions of semilinear elliptic equations with critical exponent, Differential Integral Equations, 5 (1992), 25-42.

[19] S. TERRACINI, On positive entire solutions to a class of equations with singular coefficient and critical exponent, Adv. Differential Equations, 1 (1996), 241-264.

[20] M. WILlEM, Minimax Theorems, Birkhäuser, Boston, 1996.

[21] B. XUAN, The eigenvalue problem for a singular quasilinear elliptic equation, Electron. J. Differential Equations, 16 (2004), 1-11.

[22] B. XUAN AND J. WANG, Extremal functions and best constants to an inequality involving Hardy potential and critical Sobolev exponent, Nonlinear Anal., 71 (2009), 845-859. 